\title{
A New Approach to Solve Mixed Constraint Transportation Problem Under Fuzzy Environment
}

\author{
Nidhi Joshi ${ }^{1}$, Surjeet Singh Chauhan (Gonder) ${ }^{2}$, Raghu Raja ${ }^{3}$ \\ Research Scholar, PTU, Jalandhar \\ nids_22@yahoo.co.in \\ Department of Applied Science, Chandigarh Group of Colleges, Mohali, Punjab, India \\ surjeetschauhan@yahoo.com \\ Financial Services, Accenture \\ raghu.raja001@gmail.com
}

\begin{abstract}
The present paper attempts to obtain the optimal solution for the fuzzy transportation problem with mixed constraints. In this paper, authors have proposed a new innovative approach for obtaining the optimal solution of mixed constraint fuzzy transportation problem. The method is illustrated using a numerical example and the logical steps are highlighted using a simple flowchart. As maximum transportation problems in real life have mixed constraints and these problems cannot be truly solved using general methods, so the proposed method can be applied for solving such mixed constraint fuzzy transportation problems to obtain the best optimal solutions
\end{abstract}

\section{Indexing terms/Keywords}

Optimization; Fuzzy Bi-Objective transportation problem; Ranking function; Triangular fuzzy number; Optimal solution; Improved VAM.

\section{Academic Discipline and Sub-Disciplines}

Mathematics and Fuzzy transportation problem

\section{MATHEMATICS SUBJECT CLASSIFICATION}

90C08, 90C90

\section{TYPE (METHOD/APPROACH)}

Illustration of proposed algorithm using numerical example.

\section{INTRODUCTION}

The most successful and noteworthy contribution of quantity analysis for solving business problems erstwhile in the physical distribution of products and services, is commonly referred to as transportation problems. The main objective is to minimize the cost of shipping goods from one site to another so that the requirements of each arrival area are met and every shipping site operates within its capacity.

The Transportation problem finds wide application in various industry, communication network, scheduling, planning transportation and allotment etc. The transportation problem was initially developed by Hitchcock [2] in 1941. In conventional transportation problems it was assumed that decision maker is sure about the exact and precise values of transportation cost, availability and demand of the goods. However, in real life applications, the situation and conditions are different since various parameters of the transportation problems may or may not be known precisely due to various existing uncontrollable factors. This type of imprecise and vague data may not always be well represented by random variable selected from a probability distribution. Therefore, fuzzy numbers introduced by Zadeh [1] may help in representing this kind of data. Method of fuzzy decision making, thus, is needed for real world problems. Fuzzy transportation problem (FTP) may be defined as the problem of minimizing fuzzy valued objective functions with fuzzy source and fuzzy destination parameters. At the same time in real life, most of the problems have mixed constraints but one use the FTPs for optimal solutions with equality constraints. However, the FTPs with mixed constraints are not addressed in the literature because of the rigor required to solve these problems optimally. A literature review about mixed constraint FTPs revealed no efficient method for finding its optimal solution. The More-for-less (MFL) paradox in a FTP occurs when it is possible to ship more "total goods" for less (or equal) "total fuzzy cost" while shipping the same amount or more from each origin and to each destination, keeping all shipping fuzzy costs non-negative. The mixed constraints transportation problems under crisp data have comprehensively been studied by many researchers in the past [3], [4], [5]. In 1974, Bridgen [10] considered the transportation problem with mixed constraints. Bridgen solved this problem by considering a related standard transportation problem having two additional supply points and two additional destinations. H. Isermann [9] studied transportation problem with mixed constraint and develop its solution technique in 1979. Gupta et al. [7] and Arsham [6] in 1992 obtained the more-for-less solution, for the TPs with mixed constraints by relaxing the constraints and introducing new slack variables. While obtaining the best more-for-less solution, their method is very difficult to understand since it introduces more variables and requires solving sets of complex equations. In 1998 Adlakha et al. [14] obtained a quick sufficient solution to the More-for-Less paradox in the transportation problem. Later in 2000, Adalkha and kowalski [22] provided a technical note on the procedure MFL for a more-for-less solution in transportation 
problems. In this note they modify the procedure to address issues raised by readers for their paper in which they obtained a quick sufficient solution to the More-for-Less paradox in the transportation problem, the method though efficient, does not provide specific directions in some instances. In 2001, Adalkha and Kowalski [13] developed a heuristic algorithm for solving transportation problem with mixed constraints, which was based on the theory of shadow price. In 2006, Adalkha et al. [12] provided a heuristic algorithm for solving transportation problems with mixed constraints and extend the algorithm to find a more-for-less (MFL) solution, if one exists. Adalkha et al. [11] in 2007 developed a simple heuristic algorithm to identify the demand destinations and the supply points to ship more for less in fixed charge transportation problem. Pandian and Natrajan [8] have developed fourier method for solving transportation problems with mixed constraints in 2010. They converted all the constraints in less than or equal to type constraints and eliminated variables one by one with addition of a specified constraints. In 2012, Joshi and Gupta [21] discussed the identification of more-forless paradox in the linear fractional transportation problem using objective matrix. Pandian and Anuradha [18] have introduced path method for finding a MFL optimal solution to Transportation problem in 2013. Pandian et al [15-17] also studied new method to solve transportation problem with mixed constraints in 2014. In 2014 Osuji George et al. [19] discussed an efficient statistical algorithm for computing paradox in linear transportation problem if paradox does exist. Kavitha and Anuradha [20] in 2015 proposed a new algorithm for finding the cost sensitivity analysis which determines the interval of perturbation to keep the current more-for less (MFL) optimal solution to the transportation problem remaining optimal.

In the present paper authors have attempted to find the optimal solution for fuzzy transportation problem with mixed constraints using Improved VAM method. In literature maximum work is done for the transportation problem with mixed constraints under crisp data but in real life problem there is existence of only fuzzy data. In this paper, we are using Triangular Fuzzy numbers and for the comparison of triangular fuzzy numbers we are using the ranking function.

The present paper is organized as follows: In Section-2 few basic definitions, arithmetic operations of Triangular Fuzzy numbers and method for comparing fuzzy numbers are reviewed. Section-3 is Mathematical formulation of fuzzy transportation problem with mixed constraints. In Section-4 the Proposed Algorithm and the logical representation of algorithm using flowchart are given. In Section-5 Numerical example is solved explaining the algorithm. Section-6, presents the significance and conclusion of the present study.

\section{PRELIMINARIES}

\section{Fuzzy Set}

A fuzzy set is characterized by a membership function mapping element of a domain, space or universe of discourse $X$ to the unit interval $[0,1]$ i.e. $\tilde{A}=\left\{\left(\mathrm{x}, \mu_{\tilde{A}}(\mathrm{x}) ; \mathrm{x} \in \mathrm{X}\right\}\right.$, here $\mu_{\tilde{A}}(\mathrm{x}): \mathrm{X} \rightarrow[0,1]$ is a mapping called the degree of membership function of the fuzzy set $A$ and $\mu_{\tilde{A}}(\mathrm{x})$ is called the membership value of $\mathrm{x} \varepsilon \mathrm{X}$ in the fuzzy set $\mathrm{A}$. These membership grades are often represented by real numbers ranging from $[0,1]$.

\section{Triangular Fuzzy Numbers}

It is a fuzzy number represented with three points as follows:

$\tilde{A}=\left(a_{1}, a_{2}, a_{3}\right)$, this representation is interpreted as membership functions and holds the following conditions

(i) $a_{1}$ to $a_{2}$ is increasing function.

(ii) $a_{2}$ to $a_{3}$ is decreasing function.

(iii) $a_{1} \leq a_{2} \leq a_{3}$.

$$
\mu_{A}(\mathrm{x})=\left\{\begin{array}{l}
0 \\
\frac{\left(x-a_{1}\right)}{\left(a_{2}-a_{1}\right)} \\
\frac{\left(a_{3}-x_{2}\right)}{\left(a_{3}-a_{2}\right)}
\end{array}\right.
$$

0

$$
\begin{gathered}
x<a_{1} \\
a_{1} \leq x \leq a_{2} \\
a_{2} \leq x \leq a_{3}
\end{gathered}
$$$$
x>a_{3}
$$

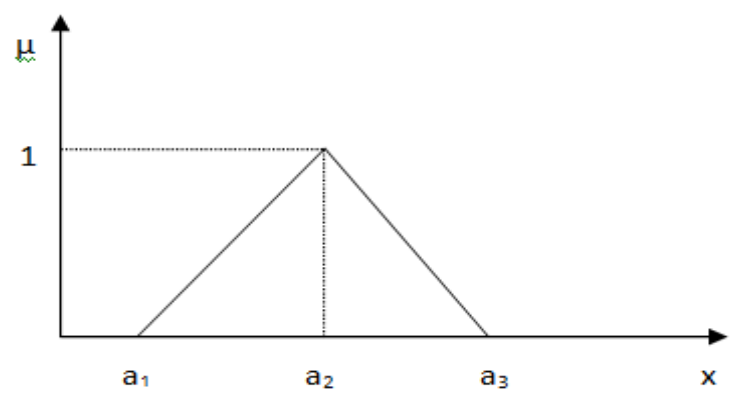

Fig 1. Triangular Fuzzy Number

\section{Properties of Triangular Fuzzy Number}

1) Triangular fuzzy number $\tilde{A}=(a, b, c)$ is said to be non negative triangular fuzzy number Iff $a-c \geq 0$ 
2) A triangular fuzzy number $\tilde{A}=(a, b, c)$ is said to be zero triangular fuzzy number Iff $a=0, b=0, c=0$.

3) Two triangular fuzzy numbers $\tilde{A} 1=(a 1, b 1, c 1)$ and $\tilde{A} 2=(a 2, b 2, c 2)$ are said to be equal lff $a 1=a 2, b 1=b 2, c 1$ $=c 2$.

\section{Arithmetic Operators for Solving Triangular Fuzzy Number}

If $\widetilde{A}=(a 1, b 1, c 1)$ and $\widetilde{B}=(a 2, b 2, c 2)$ two triangular fuzzy numbers then the arithmetic operations on $\widetilde{A}$ and $\widetilde{B}$ are as follows:

Addition $\widetilde{\mathrm{A}}+\widetilde{\mathrm{B}}=(\mathrm{a} 1+\mathrm{a} 2, \mathrm{~b} 1+\mathrm{b} 2, \mathrm{c} 1+\mathrm{c} 2)$

Subtraction $\widetilde{\mathrm{A}}-\widetilde{\mathrm{B}}=(\mathrm{a} 1-\mathrm{a} 2, \mathrm{~b} 1-\mathrm{b} 2, \mathrm{c} 1-\mathrm{c} 2)$

\section{Ranking Function}

A ranking function is defined as

$$
\mathrm{R}: F(R) \rightarrow R
$$

where $F(R)$ is set of fuzzy numbers defined on real numbers mapping each fuzzy number to real number.

In 2012, Akyar et. al. [23], gave a convenient method for ranking triangular fuzzy numbers based on their incenter and inradius. To compare triangular fuzzy numbers authors used lexicographical order by their ranks, that is, for triangular fuzzy numbers $\bar{A}$ and $\tilde{B}, \bar{A}<\bar{B}$ iff $\operatorname{Rank}(\tilde{A})<\mathrm{L}$ Rank $(\tilde{B})$, where < denotes lexicographical order.

Let $\widetilde{A}=(-0.3,-0.2,0.1)$ and $\widetilde{B}=(0.2,0.3,0.4)$. Using [44], we find

$$
\begin{aligned}
& \mathrm{r}_{\tilde{A}}=0.1633, \mathrm{r}_{\mathrm{B}} \tilde{\mathrm{B}}=0.0905, \\
& \mathrm{I}_{\tilde{A}}=(-.1195,0.1633), \mathrm{I}_{\tilde{B}}=(0.3000,0.0905), \\
& \text { and we get }
\end{aligned}
$$

$\operatorname{Rank}(\overline{\mathrm{A}})=(-0.2012,0.8367,-0.2)$,

$\operatorname{Rank}(\widetilde{\mathrm{B}})=(0.2548,0.9095,0.3)$.

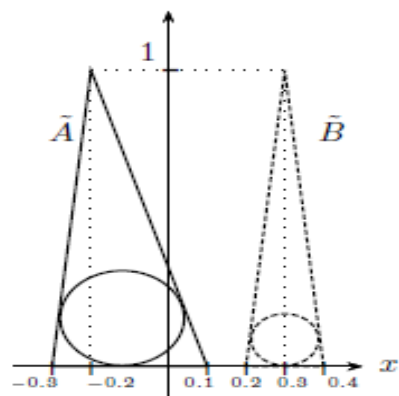

Since $(-0.2012,0.8367,-0.2)<L(0.2548,0.9095,0.3) \quad$ Triangular fuzzy numbers with their incircles in Example we conclude that $\widetilde{\mathrm{A}}<\widetilde{\mathrm{B}}$ (as shown in figure)

\section{MATHEMATICAL FORMULATION OF FUZZY TRANSPORTATION PROBLEM WITH MIXED CONSTRAINTS}

Let $m$ be the number of sources and $n$ be the number of destinations. Suppose that the cost of transporting one unit of the commodity from source $i$ to the destination $j$ is $\tilde{c}_{i j}$ Let $\tilde{a}_{i}$ be the quantity of the commodity available at source $i$ and $\tilde{b}_{j}$ be the quantity required at destination $j$. Thus $\tilde{a}_{i} \geq 0$ and $\tilde{b}_{j} \geq 0$ for all $i$ and $j$. Then the general formulation of the transportation problem with mixed constraints, as described by Pandian and Natarajan [10], is as given in table 1

Table 1: Transportation problem with mixed constraints

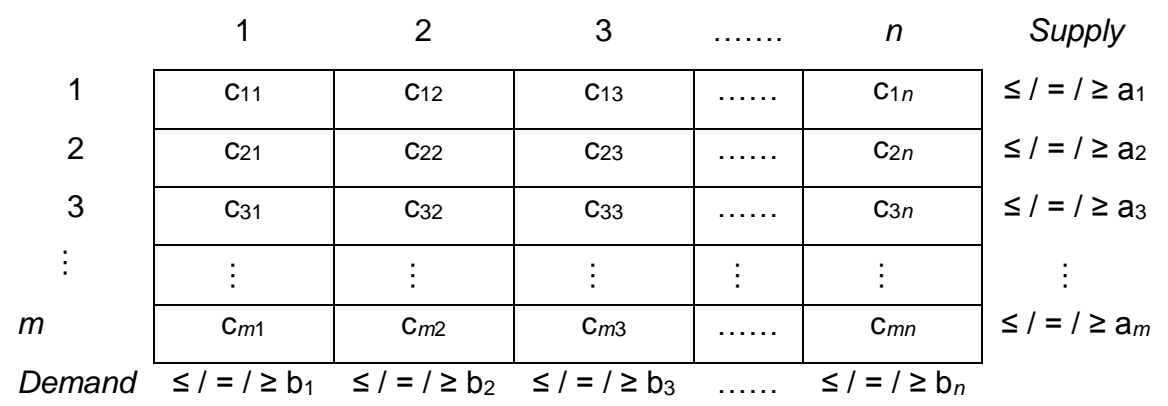

If $\tilde{x}_{i j}$ is the quantity transported from source $i$ to destination $j$ then the transportation problem is written as

Minimize $\quad Z=\sum_{i=1}^{m} \sum_{j=1}^{n} \tilde{c}_{i j} \tilde{x}_{i j}$ 
Subject to

$$
\begin{array}{cc}
\sum_{j=1}^{n} \tilde{x}_{i j} \leq\|=\| \geq \tilde{a}_{i}, \quad \forall i=1,2,3 \ldots \ldots \ldots \ldots \ldots m \\
\sum_{i=1}^{m} \tilde{x}_{i j} \leq\|=\| \geq \tilde{b}_{i}, \quad \forall j=1,2,3 \ldots \ldots \ldots \ldots \ldots n \\
\tilde{x}_{i j} \geq 0
\end{array}
$$

The above formulation represents a Linear Programming Problem (LPP) with $m \times n$ variables and $m+n$ constraints. If the LPP is small, we can solve the problem by using any simplex method, but in practical life LPP can be very large, which is difficult to solve by analytically. This type of problem can be solved very easily by using computer programming.

\section{Remark 1}

If all constraints are of equal (=) sign, then the problem becomes the transportation problem with equality constraints.

\section{ALGORITHM}

This section demonstrates the proposed algorithm. This algorithm is illustrated by the authors with the help of a numerical example in next section.

\section{PROPOSED ALGORITHM}

Step 1: Balance the given transportation problem if either (total supply>total demand) or (total supply<total demand).

Step 2: Obtain the TOC matrix. (The TOC matrix is obtained by adding the "row opportunity cost matrix" (row opportunity cost matrix: for each row, the smallest cost of that row is subtracted from each element of the same row) and the "column opportunity cost matrix" (Column opportunity cost matrix: for each column of the original transportation cost matrix the smallest cost of that column is subtracted from each element of the same column))

Step 3: Determine the penalty cost for each row and column by subtracting the lowest cell cost in the row or column from the next lowest cell cost in the same row or column.

Step 4: Select the rows or columns with the highest three penalty costs (breaking ties arbitrarily or choosing the lowestcost cell).

Step 5: Compute three transportation costs for selected three rows or columns in step 4 by assigning as many units as possible to the lowest cost square in the row or column selected.( If the assignment unit contains $\leq$ sign, then assign as lowest unit as possible. If the assignment unit is of $\geq$ sign, then assign the possible maximum value.)

We will follow the following Table to assign the supply and demand unit.

Table 2: Chart to assign Supply and Demand units

\begin{tabular}{|l|l|l|}
\hline $\operatorname{SUPPLY}\left(\tilde{a}_{i}\right)$ & DEMAND $\left(\tilde{b}_{i}\right)$ & ASSIGNMENT \\
\hline$=$ & $=$ & $\operatorname{Min}\left(\tilde{a}_{i}, \tilde{b}_{i}\right)$ \\
\hline$=$ & $\leq$ & $\operatorname{Min}\left(\tilde{a}_{i}, \tilde{b}_{i}\right)$ \\
\hline$\leq$ & $\geq$ & $\tilde{a}_{i}$ \\
\hline$\leq$ & $\leq$ & 0 \\
\hline$\leq$ & $=$ & $\operatorname{Min}\left(\tilde{a}_{i}, \tilde{b}_{i}\right)$ \\
\hline$\geq$ & $\geq$ & $\tilde{a}_{i}$ \\
\hline$\geq$ & $\geq$ & $\operatorname{Min}\left(\tilde{a}_{i}, \tilde{b}_{i}\right)$ \\
\hline$\geq$ & $=$ & $\tilde{b}_{i}$ \\
\hline
\end{tabular}

Step 6: Select minimum transportation cost of three allocations in step 5(breaking ties arbitrarily or choosing the lowestcost cell).

Step 7: Eliminate the row or column that has just been completely satisfied by the assignment just made.

Step 8: Repeat step 3-6 until all requirements have been meet.

Step 9: Compute total transportation cost for the feasible allocations using the original balanced-transportation cost matrix. 

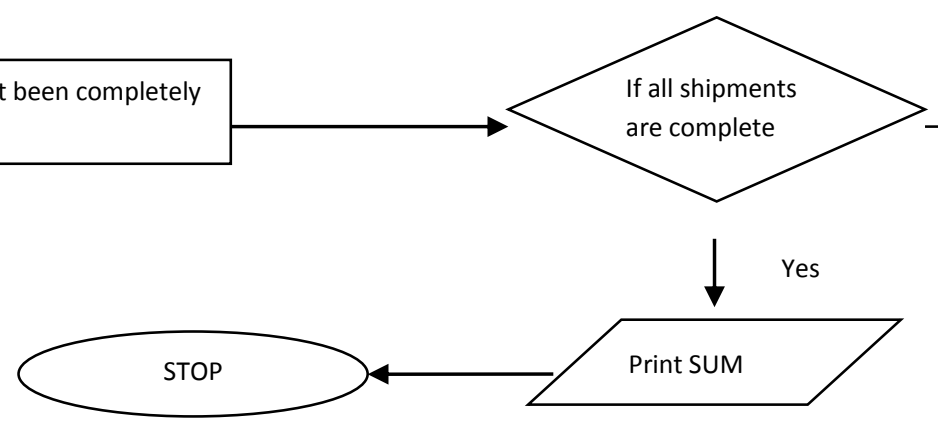

\section{NUMERICAL EXAMPLE}

Consider the following fuzzy transportation problem given below. All the data in this problem is represented by triangular fuzzy numbers. The problem is solved by the method proposed by the authors of this paper. The $X$ clothing group owns factories in three towns that distribute to four dress shops (A, B, C, D). Factory availabilities projected store demands and unit shipping costs are summarized in the table 3.

Table 3: Tableau representation of Numerical problem

\begin{tabular}{|l|l|l|l|l|l|}
\hline & A & B & C & D & Supply \\
\hline Town 1 & $(9,12,15)$ & $(1,4,7)$ & $(6,9,12)$ & $(2,5,8)$ & $=(50,55,60)$ \\
\hline Town 2 & $(5,8,11)$ & $(0,1,2)$ & $(3,6,9)$ & $(3,6,9)$ & $\geq(35,40,45)$ \\
\hline Town 3 & $(0,1,2)$ & $(1,2,3)$ & $(1,4,7)$ & $(4,7,10)$ & $\leq(25,30,35)$ \\
\hline Demand & $=(35,40,45)$ & $=(15,20,25)$ & $\leq(45,45,45)$ & $\leq(15,20,25)$ & \\
\hline
\end{tabular}

Using step 2 the TOC matrix obtained from table 3 is shown in table 4 given below

Table 4: Tableau representation of TOC Matrix

\begin{tabular}{|l|l|l|l|l|l|}
\hline & A & B & C & D & Supply \\
\hline Town 1 & $(17,19,21)$ & $(1,3,5)$ & $(10,10,10)$ & $(1,1,1)$ & $=(50,55,60)$ \\
\hline Town 2 & $(10,14,18)$ & $(0,0,0)$ & $(5,7,9)$ & $(4,6,8)$ & $\geq(35,40,45)$ \\
\hline Town 3 & $(0,0,0)$ & $(2,2,2)$ & $(1,3,5)$ & $(6,8,10)$ & $\leq(25,30,35)$ \\
\hline Demand & $=(35,40,45)$ & $=(15,20,25)$ & $\leq(45,45,45)$ & $\leq(15,20,25)$ & \\
\hline
\end{tabular}

Using Table 2 the first allocation made with the minimum transportation cost of three allocations (using step 3-6) we obtain table 5 given below

Table 5: Tableau representation of the first allocation using proposed algorithm

\begin{tabular}{|rr|r|r|r|r|}
\hline & $(17,19,21)$ & $(1,3,5)$ & $(10,10,10)$ & $(1,1,1)$ & $=(50,55,60)$ \\
\hline & $(10,14,18)$ & $(0,0,0)$ & $(5,7,9)$ & $(4,6,8)$ & $\geq(35,40,45)$ \\
\hline$(25,30,35)$ & $(0,0,0)$ & $(2,2,2)$ & $(1,3,5)$ & $(6,8,10)$ & $\leq(25,30,35)$ \\
\hline$=(35,40,45)$ & $=(15,20,25)$ & $\leq(45,45,45)$ & $\leq(15,20,25)$ & \\
\hline
\end{tabular}


Now eliminating the third row the second allocation is made similar to that as done in table 5. Second allocation is shown in table 6.

Table 6: Tableau representation of the second allocation using proposed algorithm

\begin{tabular}{|r|rr|r|r|r|}
\hline$(17,19,21)$ & $(1,3,5)$ & $(10,10,10)$ & $(1,1,1)$ & $=(50,55,60)$ \\
\hline$(10,14,18)$ & $(15,20,25)(0,0,0)$ & $(5,7,9)$ & $(4,6,8)$ & $\geq(35,40,45)$ \\
\hline$=(10,10,10)$ & $=(15,20,25)$ & $\leq(45,45,45)$ & $\leq(15,20,25)$ & \\
\hline
\end{tabular}

Similarly all other allocations can be made using the proposed algorithm and the final table obtained is shown in table 7 below.

Table 7: Tableau representation of best feasible solution

\begin{tabular}{|l|l|r|l|l|r|}
\hline & A & B & C & D & Supply \\
\hline Town 1 & \multicolumn{1}{|l|}{$(9,12,15)$} & $(1,4,7)$ & $\begin{array}{l}(35,35,35) \\
(6,9,12)\end{array}$ & \multicolumn{1}{c|}{$\begin{array}{l}(15,20,25) \\
(2,5,8)\end{array}$} \\
\hline Town 2 & $\begin{array}{l}(10,10,10) \\
(5,8,11)\end{array}$ & $\begin{array}{l}(15,20,25) \\
(0,1,2)\end{array}$ & $\begin{array}{l}(10,10,10) \\
(3,6,9)\end{array}$ & $(3,6,9)$ & $\geq(35,40,45)$ \\
\hline Town 3 & $\begin{array}{l}(25,30,35) \\
(0,1,2)\end{array}$ & $(1,2,3)$ & $(1,4,7)$ & $(4,7,10)$ & $\leq(25,30,35)$ \\
\hline Demand & $=(35,40,45)$ & $=(15,20,25)$ & $\leq(45,45,45)$ & $\leq(15,20,25)$ & \\
\hline
\end{tabular}

After applying proposed algorithm, $X_{13}=(210,315,420), X_{14}=(30,100,200), X_{21}=(50,80,110), X_{22}=(0,20,50), X_{23}=$ $(30,60,90), X_{31}=(0,30,70)$ and the best feasible solution obtained for this problem is $X_{0}=(320,605,940)$. Using step 10 the optimal solution obtained is also $X_{0}=(320,605,940)$.

\section{SIGNIFICANCE \& CONCLUSION}

1) This is a new method for solving transportation problem of More-For-Less (MFL) solution with mixed constraints.

2) The solution obtained using the proposed algorithm is very close to optimality and is among the most effective to tackle the difficult real life problems at hand. Moreover, the optimal solution obtained is fuzzy in nature, which is more realistic.

3) The algorithm does not require any deep knowledge and understanding of complex concepts like linear programming or goal and parametric programming, etc.

\section{REFRENCES}

1. Zadeh, L. A. 1965. Fuzzy sets. Information and Control, 8(3), 338-353.

2. Hitchcock, F. L. 1941. The distribution of a product from several sources to numerous localities. Journal of Math. Phys. 20, 224-230.

3. Arora, S. and Khurana, A. 2002. A paradox in an indefinite quadratic transportation problem with mixed constraints. International Journal of Management and Systems. 18(3), 301-318.

4. Lev, B. and Intrator, Y. 1977. Applications of vanishing variables methods to special structured transportation problems. Computers and Operations Research. 4, 21-26.

5. Lev, B. 1972. A non iterative algorithm for tridiagonal transportation problems and its generalization. Journal of Operations Research Society of America. 20,109-125.

6. Arsham, H. 1992. Post optimality analyses of the transportation problem. Journal of the Operational Research Society. 43,121-139.

7. Gupta, A., Khanna, S.and Puri, M. 1993. Paradoxical situation in transportation problems. Cahiers du Centre d'Etudes de RechercheOperationnell. 34, 37-49.

8. Pandian, P. and Natarajan, G. 2010. Fourier method for solving transportation problems with mixed constraints. Int. J. Contemp. Math. Sciences. 5, 28, 1385-1395.

9. Isermann, H. 1979. Solving the transportation problem with mixed constraints. Zeitschrift fur Operations Research. 26, 251-257. 
10. Brigden, M. 1974. A variant of transportation problem in which the constraints are of mixed type. Operational Research Quaterly. 25, 3, 437-445.

11. Adlakha, V., Kowalski, K., Lev, B. and Vemuganti, R.R. 2007. More-for-less algorithm for fixed-charge transportation problems. The International Journal of Management Science. 35, 1, 1-20.

12. Adlakha, V., Kowalski, K. and Lev, B. 2006. Solving transportation problems with mixed constraints. International Journal of Management Science and Engineering Management. 1, 1, 47-52.

13. Adlakha, V. and Kowalski, K. 2001. A heuristic method for more-for-less in distribution related problems. International Journal of Mathematical Education in Science and Technology. 32, 61-71.

14. Adlakha, V. and Kowalski, K. 1998. A quick sufficient solution to the More-for-Less paradox in the transportation problem. Omega. 26, 4, 541-547.

15. Pandian, P. and Natarajan, G. 2010. An Optimal More-for-Less Solution to Fuzzy Transportation Problems with Mixed Constraint. Applied Mathematical Sciences. 4, 29, 1405 - 1415.

16. Pandian, P. and Natarajan, G. 2010. A New Method for Finding an Optimal More-For-Less Solution of Transportation Problems with Mixed Constraints. Int. J. Contemp. Math. Sciences. 5, 19, $931-942$.

17. Pandian, P. and Natarajan, G. 2010. An Approach for solving Transportation Problems with Mixed Constraints. Journal of Physical Sciences. 14, 53 -61.

18. Pandian, P. and Anuradha, D. 2013. Path Method for Finding a More-For-Less Optimal Solution to Transportation Problems. International Conference on Mathematical Computer Engineering. 1, 331-337.

19. George Osuji, A., Opara, J., Anderson Nwobi, C., Onyeze, V. and Andrew Iheagwara, I. 2014. Paradox Algorithm in Application of a Linear Transportation Problem. American Journal of Applied Mathematics and Statistics. 2, 10-15.

20. Kavitha, K. and Anuradha, D. 2015. Heuristic algorithm for finding sensitivity analysis of a more for less solution to transportation problems. Global Journal of Pure and Applied Mathematics. 11, 479-485.

21. Joshi, V. D. and Gupta, N. 2012. Identifying more-for-less paradox in the linear fractional transportation problem using objective matrix. Mathematika. 28, 173-180.

22. Adlakha, V. and Kowalski, K. 2000. Technical note, A note on the procedure MFL for a more-for-less solution in transportation problems. Omega. 28, 4, 481-483.

23. Akyar, E., Akyar, H. and Duzce, S. A. 2012. A new method for ranking triangular fuzzy numbers. International Journal of Uncertainty, Fuzziness and Knowledge-based systems. 20, 5, 729-740.

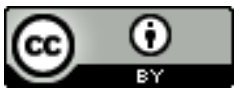

This work is licensed under a Creative Commons Attribution 4.0 International License. 